\title{
Secretor status prevents individuals from Helicobacter pylori infection
}

\author{
Arsalan Waqas Ahmad Shah ${ }^{1}$, Muhammad Asif Zeb ${ }^{2 *}$, Aman Ullah², \\ Shah Faisal Jamal ${ }^{2}$, Ebad Ullah ${ }^{1}$ and Muhammad Usman ${ }^{1}$ \\ 1. Lecturer at Seena Institute of Medical Sciences Swabi-Pakistan \\ 2. Lecturer at Institute of Paramedical Sciences, Khyber Medical University, Peshawar-Pakistan \\ *Corresponding author's email: Muhammadasif.ipms@kmu.edu.pk \\ Citation \\ Arsalan Waqas Ahmad Shah, Muhammad Asif Zeb, Aman Ullah, Shah Faisal Jamal, Ebad Ullah and Muhammad \\ Usman. Secretor status prevents individuals from Helicobacter pylori infection. Pure and Applied Biology. Vol. \\ 10, Issue 2, pp503-508. http://dx.doi.org/10.19045/bspab.2021.100053
}

\begin{tabular}{llll}
\hline \hline Received: 13/04/2020 & Revised: 22/10/2020 & Accepted: 26/10/2020 & Online First: 02/11/2020 \\
\hline \hline
\end{tabular}

\section{Abstract}

ABO antigens are not only present on the surface of red blood cells but are also secreted in body fluids. Individuals who can secrete these antigens in body fluids are called secretors while those who cannot are known as non-secretors. As compared to secretor non-secretors are at high risk to different bacterial, heart and autoimmune diseases but it is not clear that secretor status has an association with Helicobacter pylori. Therefore, this study was conducted to determine the secretor and non-secretor status in Helicobacter pylori infected patients. This cross-sectional study was carried out at Institute of Paramedical Sciences, Khyber Medical University Peshawar. A total of 192 samples were included in this study, 96 from Helicobacter pylori infected patients and 96 as a control group. Saliva samples were taken for the determination of secretor and non-secretor status by using Absorption inhibition method. Blood samples were obtained for ABO blood grouping and Helicobacter pylori determination. Out of 96 Helicobacter pylori infected patients, 36 (37.5\%) were found as secretors and $60(62.5 \%)$ were found as non-secretors while in normal individuals, secretors were 67 (69.8\%) and nonsecretors were $29(30.2 \%)$. In H. pylori infected patients, classification of various ABH blood groups like A, B, AB and $\mathrm{O}$ were $24(25 \%), 33(34.3 \%), 18(18.8 \%)$ and $21(21.9 \%)$ respectively. In normal individuals' the frequency of ABO was $28(29.2 \%)$ of A, $28(29.2 \%)$ of $\mathrm{B}, 8(8.3 \%)$ of $\mathrm{AB}$ and $32(33.3 \%)$ of $\mathrm{O}$ blood groups respectively. It is concluded that Helicobacter pylori infected patients have lowest secretor and highest non-secretors, because $\mathrm{ABH}$ antigens present in the body fluids of secretor show resistance to Helicobacter pylori infection. Moreover, non-secretors are more susceptible to bacterial infections. And it is known that Helicobacter pylori is a bacterium which can cause bacterial infections like gastric ulcer and duodenal ulcer. While normal individuals have highest secretor and lowest non-secretor status.

Keywords: ABO blood groups; Body fluids; Helicobacter pylori; Non-secretor

\section{Introduction}

Blood group is one of the basic factors for the recognition of an individual or for the determination of suspected unidentified individuals. $\mathrm{A}, \mathrm{B}, \mathrm{AB}$ and $\mathrm{O}$ are the four major types of $\mathrm{ABO}$ blood group system [1]. In 1926, it was observed that apart from the surface of red blood cells, (RBCs) ABO antigens are also present in soluble form in body fluids like saliva, tear, milk, sweat, semen, urine and amniotic fluids. In 1930, Putkonen classified the individuals into secretor or non-secretor on the basis of their genetic ability to secrete ABO blood group 
antigens in their body fluids such as saliva [2].

Around the globe, $80 \%$ of the people are secretor and $20 \%$ are non-secretors. Secretor status might be mismatched in various populations due to some geographical and racial factors [3]. In India, percentage of secretor and non-secretor are $72.4 \%$ and $27.6 \%$ respectively [4]. While in Karachi Pakistan, the frequency of secretor is $64 \%$ and non-secretor is $36 \%$ [5].

$\mathrm{ABH}$ secretor saliva includes extra carbohydrate compounds in the mucin that accumulate some bacteria and reduce their action. As compared to secretors, nonsecretors are more prone to diseases such as esophageal cancer, mouth diseases and epithelial dysplasia [6]. During blood grouping, non-Hodgkin lymphomas and many malignancies like leukemias change the erythrocyte antigens and represent weaker reactivity therefore, saliva is investigated for the determination of patient blood group [7].

Non-secretors are at high risk of thrombotic and heart diseases because of higher plasma concentration of Von Willebrand factor (VWF) and factor VIII. ABO non-secretor has increased chances of duodenal ulcer, persistent candida infections and frequent urinary tract infections. Besides these diseases, non-secretors also have a higher prevalence of many autoimmune diseases like ankylosing spondylitis, grave's disease, psoriatic multiple sclerosis, reactive arthritis and Sjogren's syndrome [8]. Persons who do not have the ability to secrete $\mathrm{ABO}$ antigens in gastrointestinal mucosa have been linked with peptic ulcer, gastric malignancy and pernicious anemia [9].

One of the most chronic occurring bacterial infection is $H$. pylori which affects approximately more than half of the population around the globe with high geographic deviation [10]. The risk of $\mathrm{H}$. pylori in developed countries is < $40 \%$ while it is much higher in developing countries i.e. $>80 \%[11,12]$. In Pakistan, about $50-90 \% \mathrm{H}$. pylori have been reported [13-17].

Although some studies have determined the relationships of $\mathrm{ABO}$ and secretor/nonsecretor phenotypes with $H$. pylori infection, yet it is unclear that an association is present. According to Nikanne et al., there is no association between secretor phenotype and Helicobacter pylori serology by studying 271 blood donors [18]. Chesner et al., analysed 185 patients with dyspepsia but not peptic ulcer in order to find out relationship between secretor and $\mathrm{H}$. pylori status and at the end he found no association [19].

As there is no clear evidence that secretor and $\mathrm{H}$. pylori are linked to each other. Moreover, formerly there was no independently study carried out on $\mathrm{H}$. pylori infected patients for the determination of secretor status in Peshawar Pakistan. Therefore, this study was conducted to compare secretor and non-secretor status among $H$. pylori infected patients.

\section{Materials and Methods}

This cross-sectional comparative study was carried out (February 2019 to July 2019) at Khyber Medical University (KMU) Peshawar in the Institute of Paramedical Sciences (IPMS) after the approval from the Undergraduate research committee of IPMS. Convenience sampling technique was used in this study. A total of 192 individuals including 96 Helicobacter pylori infected patients and 96 normal individuals were selected as control. All those patients who were infected with Helicobacter Pylori were included in this study. Individuals who had chronic diseases like Hepatitis B Virus (HBV), Hepatitis C Virus (HCV), Human Immunodeficiency Virus (HIV), mentally retarded and active oral cavity disease patients were excluded from the study. Informed consent was taken from all the individuals. Two millilitre saliva samples was collected from all the participants in a disposable sterilized container and three millilitre venous blood 
sample in Ethylenediamine tetra acetic acid (EDTA) tube.

Individuals were asked to wash their mouth properly with distilled water. From the collection bottle saliva was shifted into sterile test tube and was kept in water bath for 10 minutes at $90^{\circ} \mathrm{C}$ to denature bacterial enzymes. Sample tube was placed at room temperature for 10 minutes and then centrifuged at 3000 rpm for 10 minutes. Supernatant was transferred from each tube to another test tube and was used for the Absorption inhibition method.

Tubes were labelled as A, B, AB, O and control respectively. One drop of processed saliva was added to each tube while in control tube one drop of normal saline was added. Moreover, one drop of diluted antisera was added to the corresponding tubes. Tubes were mixed and incubate at $37^{\circ} \mathrm{C}$ for 10 minutes. After incubation, tubes were cooled at room temperature for 10 minutes. Further, single drop of $3 \%$ red cells suspension were added to all tubes. After 10 minutes re-shake the tubes and checked for agglutination macroscopically. For confirmation of agglutination the slide was examined microscopically. Secretor was identified by the absence of agglutination while non-secretor was identified by the presence of agglutination [20]. From venous blood, forward blood grouping was carried out by means of slide agglutination method. For $\mathrm{H}$ pylori determination, Immunochromatography technique (ICT) was used. SPSS-16 was used for statistical analysis and cross tabulation. Pearson chi square test was applied and found statistically significant $(\mathrm{X} 2=0.00)$.

As we used convenience sampling technique, the frequency and prevalence of H. pylori patient are greater in males compared to females in Peshawar, which was the limitation of this study.

\section{Results}

For the identification of $\mathrm{ABO}$ secretor and non-secretor status, total numbers of 192 samples were analysed. Among them 96 were taken as $\mathrm{H}$. pylori infected patients and 96 were taken as normal individuals. Out of 96, H. pylori infected patients, 36 $(37.5 \%)$ were found as secretors and 60 $(62.5 \%)$ were found as non-secretors. While in 96 normal individuals, 67 (69.8\%) were found as secretors and 29 (30.2\%) were found as non-secretors (Table 1).

Table 1. H. pylori Infected patients

\begin{tabular}{|c|c|c|c|}
\hline Variables & Total Cases & Secretors & Non-secretors \\
\hline H. Pylori Patients & 96 & $36(37.5 \%)$ & $60(62.5 \%)$ \\
\hline Normal Individuals & 96 & $67(69.8 \%)$ & $29(30.2 \%)$ \\
\hline
\end{tabular}

Based on gender, out of $96 \mathrm{H}$. pylori infected patients, $75(78.1 \%)$ were male in which secretors and non-secretors were 33 (44\%) and $42(56 \%)$ respectively. Whereas in $21(21.9 \%) \mathrm{H}$. pylori infected females, secretors and non-secretors were $8(38.1 \%)$ and $13(61.9 \%)$ respectively (Table 2$)$. Based on gender among the normal individuals, $80(83.33 \%)$ were male in which the secretors and non-secretors were $56(70 \%)$ and $24(30 \%)$ respectively. Likewise, in $16(16.67 \%)$ females the secretors and non-secretors were 11 $(68.75 \%)$ and $5(31.25 \%)$ respectively (Table 2).

Table 2. Gender wise distribution of secretors and non-Secretors in H. pylori patients and normal individuals

\begin{tabular}{|c|c|c|c|c|}
\hline \multirow{2}{*}{ Variables } & \multicolumn{2}{|c|}{ H. Pylori Patients } & \multicolumn{2}{c|}{ Normal Individuals } \\
\cline { 2 - 5 } & Male & Female & Male & Female \\
\hline Frequency & $75(78.1 \%)$ & $21(21.9 \%)$ & $80(83.3 \%)$ & $16(16.7 \%)$ \\
\hline Secretor & $33(44 \%)$ & $8(38.1 \%)$ & $56(70 \%)$ & $11(68.8 \%)$ \\
\hline Non-Secretor & $42(56 \%)$ & $13(61.9 \%)$ & $24(30 \%)$ & $5(31.2 \%)$ \\
\hline
\end{tabular}


Based on $\mathrm{Rh}$ group system of $\mathrm{H}$. pylori infected patients, out of $92(95.8 \%) \mathrm{Rh}-$ positive members secretors were 40 $(43.5 \%)$ and non-secretors were 52 $(56.5 \%)$. Whereas in $4(4.2 \%) \mathrm{Rh}$-negative members number of both secretors and nonsecretors were same i.e. $2(50 \%)$ as mentioned in (Table 3). Based on Rh group system of normal individuals, out of 91 (94.8\%) Rh-positive members secretors were $65(71.4 \%)$ and non-secretors were 26 (28.6\%). While in $5(5.2 \%) \mathrm{Rh}$-negative members number of secretors and nonsecretors were $2(40 \%)$ and $3(60 \%)$ respectively (Table 3 ).

Table 3. Rh group wise distribution of secretors and non-secretors in $H$. pylori patients and normal individuals

\begin{tabular}{|c|c|c|c|c|}
\hline \multirow{2}{*}{ Variables } & \multicolumn{2}{|c|}{ H. Pylori Patients } & \multicolumn{2}{c|}{ Normal Individuals } \\
\cline { 2 - 5 } & Rh-Positive & Rh-Negative & Rh-Positive & Rh-Negative \\
\hline Frequency & $92(95.8 \%)$ & $4(4.2 \%)$ & $91(94.8 \%)$ & $5(5.2 \%)$ \\
\hline Secretor & $40(43.5 \%)$ & $2(50 \%)$ & $65(71.4 \%)$ & $2(40 \%)$ \\
\hline Non-Secretor & $52(56.5 \%)$ & $2(50 \%)$ & $26(28.6 \%)$ & $3(60 \%)$ \\
\hline
\end{tabular}

\section{Discussion}

To the best of our knowledge in the past there was no study conducted on $\mathrm{H}$. pylori infected patients for the determination of secretor and non-secretor status in Peshawar Pakistan. As compared to normal individuals $\mathrm{H}$. pylori infected patients have low frequency of secretors and high frequency of non-secretors because nonsecretors are more susceptible to bacterial infections. H. pylori is a bacteria that causes infections like gastric ulcer and duodenal ulcer $[21,22]$. Therefore the percentage of non-secretor is more in $\mathrm{H}$. pylori infected patients than normal individuals. Whereas the secretor which contain $\mathrm{ABH}$ antigens in body fluids like saliva and gastrointestinal mucosa show resistance to $\mathrm{H}$. pylori infection because these antigens make members of human mucosal innate immune system [23]. Our study is comparable to the study conducted by Saboor et al., with that of normal individuals as they conducted study on normal individuals [5].

In contrast, the findings of Rashmi et al., reported $99 \%$ secretors and $1 \%$ nonsecretors. As blood group system are genetically controlled and inherited. Rashmi conducted his study in families therefore, the percentage of secretor was increased as compared to our study [24]. In our study in $\mathrm{H}$. pylori infected patients, the frequency of $\mathrm{ABO}$ was $24(25 \%)$ of $\mathrm{A}$,
$33(34.4 \%)$ of $\mathrm{B}, 18(18.8 \%)$ of $\mathrm{AB}$ and $21(21.9 \%)$ of O blood groups respectively. It shows that in our locality participants with B blood group present in elevated frequency and participants with $\mathrm{AB}$ blood group present in lowest frequency in $\mathrm{H}$. pylori infected patients as compare to other blood groups. In normal individuals the classification of various $\mathrm{ABH}$ blood groups like $\mathrm{A}, \mathrm{B}, \mathrm{AB}$ and $\mathrm{O}$ were $28(29.2 \%)$, $28(29.2 \%), \quad 9(9.4 \%)$ and $31(32.3 \%)$ respectively. Individual with $\mathrm{O}$ blood group present in highest percentage and individuals with $\mathrm{AB}$ blood group present in lowest percentage as compared to other blood groups. Ali et al., carried study on various populations of Pakistan and verified the above findings [25].

In our study in $\mathrm{H}$. pylori infected patients, $92(95.83 \%)$ were found as Rh-positive members and 4(4.17\%) were found as Rhnegative members. In Rh-positive $\mathrm{H}$. pylori infected patients $40(43.48 \%)$ were found as secretors and $52(56.52 \%)$ were found as non-secretors. While in Rh-negative individuals' secretors were $2(50 \%)$ and the non-secretors were also $2(50 \%)$. In normal individuals, 91 (94.8\%) were found as Rhpositive members and $5(5.2 \%)$ were found as Rh-negative members. In Rh-positive normal individuals $65(71.43 \%)$ were found as secretor and $26(28.57 \%)$ were found as non-secretor. The study of Jiff et al., show 
similarity to our study of normal individuals. While in Rh-negative normal individual's 2(40\%) were found as secretor and 3(60\%) were found as non-secretor [3].

\section{Conclusion}

It is concluded that non-secretor individuals are more prone to $\mathrm{H}$ pylori infection while individuals with secretor status show resistance to $\mathrm{H}$ pylori infection. Further research is required by correlating the patient status i.e. severity of the disease and clinical investigation and endoscopy to accurately correlate the secretion status with $\mathrm{H}$. pylori infection. This study was conducted as a general statistical correlation between $\mathrm{H}$. pylori and $\mathrm{ABO}$ antigen secretion, in order to find out more accurate results that $\mathrm{ABO}$ antigen secretion and $\mathrm{H}$. pylori were independent risk factors for gastro-duodenal disease, logistic regression analysis on the endoscopy performed on dyspeptic symptoms patient's data with proper exclusion of confounding variables. As medical science is moving toward molecular level, so on molecular basis investigation and research is required to find out the gene expression or their specific $H$. pylori specific epitopes expression correlation with that of $\mathrm{ABO}$ antigen secretion.

\section{Authors' contributions}

Conceived, planned and supervised the project: MA Zeb, A Ullah \& SH Jamal, Performed the experiment: AWA Shah, M Usman \& E Ullah, Contributed reagents/ materials/ analysis/ tools: AWA Shah, M Usman \& E Ullah, Wrote the paper: AWA Shah, MA Zeb, A Ullah, \& SF Jamal, Helped in proofreading of the manuscript: AWA Shah, MA Zeb, A Ullah \& SF Jamal.

\section{References}

1. Daniels G (1995). ABO, Hh and Lewis systems. Human Blood Groups: Blackwell Sci 7-67.

2. Daniels G (2002). ABO, Hh and lewis systems. In: Daniels G. Human Blood Groups. In: Oxford (UK): Blackwell Sci.

3. Jaff MS (2010). Higher frequency of secretor phenotype in $\mathrm{O}$ blood group- its benefits in prevention and/or treatment of some diseases. Inter $J$ Nanomed 5: 901.

4. Poonam W (2017). To find out the prevalence of abh secretor and nonsecretor status on the basis of saliva test and it's clinical significance among the population of greater gwalior region, pp. 201.

5. Saboor M, Ullah A, Qamar K \& Mir A (2014). Frequency of ABH secretors and non secretors: A cross sectional study in Karachi. Pak J Med Sci 30(1): 189.

6. Campi C, Escovich L, Moren A, Racca L, Racca A \& Cotoruelo C (2012). Expression of the gene encoding secretor type galactoside $2-\alpha-\mathrm{L}-$ Fucosyltransferase (FUT2) and $\mathrm{ABH}$ antigens in patients with oral lesions. Med Oral Patol Oral Cir Bucal 17(1): 63-68.

7. Akhter S, Kibria G, Akhter N, Habibullah M, Islam S, \& Zakariah M (2011). ABO and Lewis blood grouping with $\mathrm{ABH}$ secretor and nonsecretor status: A cross sectional study in Dhaka. Faridpur Med Coll J 6(1): 38-40.

8. D-Adamo PJ, \& Kelly GS (2001). Metabolic and immunologic consequences of $\mathrm{ABH}$ secretor and Lewis subtype status. Altern Med Rev 6(4): 390-405.

9. Suadicani $P$, Hein $H$, \& Gyntelberg $F$ (1999). Genetic and life-style determinants of peptic ulcer: A study of 3387 men aged 54 to 74 years: The Copenhagen Male Study. Scand J Gastroenterol 34(1): 12-17.

10. Hooi JKY, Lai WY, Ng WK, Suen MMY, Underwood FE, Tanyingoh D, Malfertheiner P, Graham DY, Wong VWS \& Wu JCY (2017). Global Prevalence of Helicobacter pylori Infection: Systematic Review and Meta-Analysis. Gastroenterol 153: 420-429.

11. Brown LM, Thomas TL, Ma Jl, Chang YS, You WC, Liu WD, \& Gail MH 
(2002). Helicobacter pylori infection in rural China: demographic, lifestyle and environmental factors. Inter $J$ Epidemiol 31(3): 638-645.

12. Sinha SK, Martin B, Sargent M, McConnell JP, \& Bernstein CN (2002). Age at acquisition of Helicobacter pylori in a pediatric Canadian First Nations population. Helicobacter 7(2): 76-85.

13. Hassan SR, \& Abbas Z (2007). Presence of Helicobacter pylori in dyspeptic patients with endoscopically normal stomach. Pak J Med Sci 23(3): 335.

14. Nizami SQ, Bhutta ZA, Weaver L, \& Preston T (2005). Helicobacter pylori colonization in infants in a periurban community in Karachi, Pakistan. $J$ Pediatr Gastroenterol Nutr 41(2): 191194.

15. Abbas Z, Jafri W, Khan AH, \& Shah MA (1998). Prevalence of Helicobacter pylori antibodies in endoscopy personnel and non-medical volunteers of Karachi. J Pak Med Assoc 48: 201-202.

16. Ahmad T, Sohail K, Rizwan M, Mukhtar M, Bilal R, \& Khanum A (2009). Prevalence of Helicobacter pylori pathogenicity-associated cagA and vacA genotypes among Pakistani dyspeptic patients. FEMS Immunol Med Mic 55(1): 34-38.

17. Mehmood K, Hameed Z, Shoukat S, Hasan F, Alam AY, Hameed A, \& Nadir A (2011). Predictors of depression in patients presenting with dyspeptic symptoms in a GI clinic. $J$ Ayub Med Coll Abbottabad 23(4): 4952.

18. Höök-Nikanne J, Sistonen $\mathrm{P}, \quad \&$ Kosunen T (1990). Effect of ABO blood group and secretor status on the frequency of Helicobacter pylori antibodies. Scand. J Gastroenterol 25(8): 815-818.

19. Chesner I, Nicholson G, FEREYDOUN A, Elliott $\mathrm{T}$, \& Langman M (1992). Predisposition to gastric antral infection by Helicobacter pylori: an investigation of any association with ABO or Lewis blood group and secretor status. Eur $J$ Gastroenterol Hepatol 4(5): 377-379.

20. Sen MP, Vanishree M, Hunasgi S, Surekha R, Koneru A, \& Manvikar V (2015). A comparison of absorption inhibition and absorption elution methods for estimation of ABO blood groups in saliva. J Med Radiol Pathol Surg 1(1): 1-4.

21. Blackwell C (1989). The role of ABO blood groups and secretor status in host defences. FEMS Microbiol Immunol 1(6-7): 341-349.

22. Clarke CA, Edwards JW, Haddock DR, Howel-Evans A, McConnell RB, \& Sheppard P (1956). ABO blood groups and secretor character in duodenal ulcer. BMJ 2(4995): 725.

23. Linden S, Mahdavi J, Semino-Mora C, Olsen C, Carlstedt 1, Borén T, \& Dubois A (2008). Role of ABO secretor status in mucosal innate immunity and $\mathrm{H}$. pylori infection. PLOS Pathog 4(1): e2.

24. Metgud R, Khajuria N, Mamta, Ramesh G (2016). Evaluation of the secretor status of abo blood group antigens in saliva among southern rajasthan population using absorption inhibition method. J Clin Diagnostic Res 10(2).

25. Al Ghamdi AS (2009). Association between ABO blood groups and severity of chronic periodontitis. $J$ King Abdulaziz Univ Med Sci 16(3): 31-41. 